\title{
Optimal Time Evolution for Hermitian and Non-Hermitian Hamiltonians
}

\author{
Carl M. Bender* and Dorje C. Brody ${ }^{\dagger}$ \\ *Department of Physics, Washington University, St. Louis MO 63130, USA \\ ${ }^{\dagger}$ Department of Mathematics, Imperial College London, London SW7 2AZ, UK \\ The shortest path between two truths in the real domain passes through the \\ complex domain. - Jacques Hadamard, The Mathematical Intelligencer 13 (1991)
}

Consider the set of all Hamiltonians whose largest and smallest energy eigenvalues, $E_{\max }$ and $E_{\min }$, differ by a fixed energy $\omega$. Given two quantum states, an initial state $\left|\psi_{I}\right\rangle$ and a final state $\left|\psi_{F}\right\rangle$, there exist many Hamiltonians $H$ belonging to this set under which $\left|\psi_{I}\right\rangle$ evolves in time into $\left|\psi_{F}\right\rangle$. Which Hamiltonian transforms the initial state to the final state in the least possible time $\tau$ ? For Hermitian Hamiltonians, $\tau$ has a nonzero lower bound. However, among complex non-Hermitian PT-symmetric Hamiltonians satisfying the same energy constraint, $\tau$ can be made arbitrarily small without violating the time-energy uncertainty principle. The minimum value of $\tau$ can be made arbitrarily small because for $P T$-symmetric Hamiltonians the evolution path from the vector $\left|\psi_{I}\right\rangle$ to the vector $\left|\psi_{F}\right\rangle$, as measured using the Hilbert-space metric appropriate for this theory, can be made arbitrarily short. The mechanism described here resembles the effect in general relativity in which two space-time points can be made arbitrarily close if they are connected by a wormhole. This result may have applications in quantum computing.

\section{INTRODUCTION}

Interest in optimal time evolution dates back to the end of the seventeenth century, when the famous brachistochrone problem was solved almost simultaneously by Newton, Leibniz, l'Hôpital, and Jacob and Johann Bernoulli. The word brachistochrone is derived from Greek and means shortest time (of flight). The classical brachistochrone problem is stated as follows: A bead slides down a frictionless wire from point $A$ to point $B$ in a homogeneous gravitational field. What is the shape of the wire that minimises the time of flight of the bead? The solution to this problem is that the optimal (fastest) time evolution is achieved when the wire takes the shape of a cycloid, which is the curve that is traced out by a point on a wheel that is rolling on flat ground.

In the past few years there has been much interest in the quantum brachistochrone problem, which is formulated in a somewhat similar fashion: Consider two fixed quantum states, an initial state $\left|\psi_{I}\right\rangle$ and a final state $\left|\psi_{F}\right\rangle$ in a Hilbert space. We then consider the set of all Hamiltonians satisfying the energy constraint that the difference between the largest and smallest eigenvalues is a fixed energy $\omega: E_{\max }-E_{\min }=\omega$. Some of the Hamiltonians in this set allow the initial state $\left|\psi_{I}\right\rangle$ to evolve into the final state $\left|\psi_{F}\right\rangle$ in time $t$ :

$$
\left|\psi_{F}\right\rangle=\mathrm{e}^{-\mathrm{i} H t / \hbar}\left|\psi_{I}\right\rangle
$$


The quantum brachistochrone problem is to find the optimal Hamiltonian; that is, the Hamiltonian that accomplishes this evolution in the shortest possible time, which we denote by $\tau$.

In this chapter we show that for Hermitian Hamiltonians the shortest evolution time $\tau$ is a nonzero quantity whose size depends on the Hilbert-space distance between the fixed initial and final state vectors. However, for complex non-Hermitian Hamiltonians, the value of $\tau$ can be made arbitrarily small. Thus, non-Hermitian Hamiltonians permit arbitrarily fast time evolution.

Of course, a non-Hermitian Hamiltonian may be physically unrealistic because it may possess complex eigenvalues and/or it may generate nonunitary time evolution; that is, time evolution in which probability is not conserved. However, there is a special class of non-Hermitian Hamiltonians that are PT symmetric; that is, Hamiltonians that are invariant under combined space and time reflection. Although such Hamiltonians are not Hermitian in the Dirac sense, they do have entirely real spectra and give rise to unitary time evolution. Thus, such Hamiltonians define consistent and acceptable theories of quantum mechanics. We show in this chapter that if we use Hamiltonians of this type to solve the quantum brachistochrone problem, we can achieve arbitrarily fast time evolution without violating any principles of quantum mechanics. Thus, if it were possible to implement fasterthan-Hermitian time evolution, then non-Hermitian Hamiltonians might have important applications in quantum computing.

This chapter is organised as follows: In Sec. [I] we introduce and describe PT quantum mechanics and explain how a Hamiltonian that is not Dirac Hermitian can still define a consistent theory of quantum mechanics. Then in Sec. [III we explain why complex classical mechanics allows for faster-than-conventional time evolution. In Sec. IV] we discuss the quantum brachistochrone for Hermitian Hamiltonians. Then, in Sec. V we extend the discussion in Sec. [V] to Hamiltonians that are not Dirac Hermitian. In Sec. VI] we explain how it might be possible for a complex Hamiltonian to achieve faster-than-Hermitian time evolution.

\section{PT QUANTUM MECHANICS}

Based on the training that one receives in a traditional quantum mechanics course, one would expect a theory defined by a non-Hermitian Hamiltonian to be physically unacceptable because the energy levels would most likely be complex and the time evolution would most likely be nonunitary (not probability-conserving). However, theories defined by a special class of non-Hermitian Hamiltonians called PT-symmetric Hamiltonians can have positive real energy levels and can exhibit unitary time evolution. Such theories are consistent quantum theories. It may be possible to distinguish these theories experimentally from theories defined by Hermitian Hamiltonians because, in principle, non-Hermitian Hamiltonians can be used to generate arbitrarily fast time evolution.

We use the following terminology in this chapter: By Hermitian, we mean Dirac Hermitian, where the Dirac Hermitian adjoint symbol ${ }^{\dagger}$ represents combined matrix transposition and complex conjugation. The parity operator $P$ performs spatial reflection and thus in quantum mechanics it changes the sign of the position operator $x$ and the momentum operator $p: P x P=-x$ and $P p P=-p$. Because the parity operator $P$ is a reflection operator, its square is the unit operator: $P^{2}=\mathbb{1}$. The time-reversal operator $T$ performs the time reflection $t \rightarrow-t$, and thus it changes the sign of the momentum operator $p, T p T=-p$, 
but it leaves the position operator invariant: $T x T=x$. The square of $T$ is the unit operator $T^{2}=\mathbb{1}$. We require that the operators $P$ and $T$ individually leave invariant the fundamental Heisenberg algebra of quantum mechanics $[x, p]=\mathrm{i}$. Thus, while $P$ is a linear operator, we see that $T$ must perform complex conjugation $T z T=z^{*}$, and hence $T$ is an antilinear operator. [62]

The first class of $P T$-symmetric quantum-mechanical Hamiltonians was introduced in 1998 [6]. Since then there have been many papers on this subject by a wide range of authors. There have also been three recent review articles [4, 5, 29]. In Ref. [6] it was discovered that even if a Hamiltonian is not Hermitian, its energy levels can be all real and positive so long as the eigenfunctions are symmetric under $P T$ reflection.

These new kinds of Hamiltonians are obtained by deforming ordinary Hermitian Hamiltonians into the complex domain. The original class of $P T$-symmetric Hamiltonians that was proposed in Ref. [6] has the form

$$
H=p^{2}+x^{2}(\mathrm{i} x)^{\epsilon} \quad(\epsilon>0),
$$

where $\epsilon$ is a real deformation parameter. Two particularly interesting special cases are obtained by setting $\epsilon=1$ to get $H=p^{2}+\mathrm{i} x^{3}$ and by setting $\epsilon=2$ to get $H=p^{2}-x^{4}$. Surprisingly, these Hamiltonians have real, positive, discrete energy levels even though the potential for $\epsilon=1$ is imaginary and the potential for $\epsilon=2$ is upside-down. The first complete proof of spectral reality and positivity for $H$ in (2) was given by Dorey et al. in Ref. [27, 28].

The philosophical background of $P T$ quantum mechanics is simply this: One of the axioms of quantum mechanics requires that the Hamiltonian $H$ be Dirac Hermitian. This axiom is distinct from all other quantum-mechanical axioms because it is mathematical rather than physical in character. The other axioms of quantum mechanics are stated in physical terms; these other axioms require locality, causality, stability and uniqueness of the vacuum state, conservation of probability, Lorentz invariance, and so on. The condition of Dirac Hermiticity $H=H^{\dagger}$ is mathematical, but the condition of $P T$ symmetry $H=H^{P T}=$ $(P T) H(P T)$ (space-time reflection symmetry) is physical because $P$ and $T$ are elements of the Lorentz group.

The spectrum of $H$ in (2) is real, which poses the question of whether this Hamiltonian specifies a quantum-mechanical theory. That is, is the theory specified by $H$ associated with a Hilbert space endowed with a positive inner product and does $H$ specify unitary (norm-preserving) time evolution? The answer to these questions is yes. Positivity of the inner product and unitary time evolution was established in Ref. [11, 12] for quantummechanical systems having an unbroken $P T$ symmetry (an analogous result was obtained by Mostafazadeh in Ref. [50]) and in Ref. [13] for quantum field theory.

To demonstrate that the theory specified by the $H$ in (2) is a quantum-mechanical theory, we construct a linear operator $C$ that satisfies the three simultaneous algebraic equations [11]: $C^{2}=\mathbb{1},[C, P T]=0$, and $[C, H]=0$. Using $C$, which in quantum field theory is a Lorentz scalar [8], we can then construct the appropriate inner product for a $P T$-symmetric Hamiltonian: $\langle a \mid b\rangle \equiv a^{C P T} \cdot b$. This inner product, which uses the $C P T$ adjoint, has a strictly positive norm: $\langle a \mid a\rangle>0$. Because $H$ commutes with both $P T$ and $C, H$ is self-adjoint with respect to $C P T$ conjugation. Also, the time-evolution operator $\mathrm{e}^{-\mathrm{i} H t / \hbar}$ is unitary with respect to $C P T$ conjugation. Note that the Hilbert space and the $C P T$ inner product are dynamically determined by the Hamiltonian itself.

We have explained why a $P T$-symmetric Hamiltonian gives rise to a unitary theory, but in doing so we raise the question of whether $P T$-symmetric Hamiltonians are useful. The 
answer to this question is simply that PT-symmetric Hamiltonians have already been useful in many areas of physics. For example, in $1959 \mathrm{Wu}$ showed that the ground state of a Bose system of hard spheres is described by a non-Hermitian Hamiltonian [60]. Wu found that the ground-state energy of this system is real and he conjectured that all of the energy levels were real. Hollowood showed that the non-Hermitian Hamiltonian for a complex Toda lattice has real energy levels [41]. Cubic non-Hermitian Hamiltonians of the form $H=p^{2}+\mathrm{i} x^{3}$ (and also cubic quantum field theories having an imaginary self-coupling term) arise in studies of the Lee-Yang edge singularity [24, 25, 31, 61] and in various Reggeon field-theory models [22, 38, 39]. In all of these cases a non-Hermitian Hamiltonian having a real spectrum appeared mysterious at the time, but now the explanation is clear: In every case the nonHermitian Hamiltonian is PT symmetric. Hamiltonians having PT symmetry have also been used to describe magnetohydrodynamic systems [34, 37] and to study nondissipative time-dependent systems interacting with electromagnetic fields [30].

An important application of PT quantum mechanics is in the revitalisation of theories that have been thought to be dead because they appear to have ghosts. Ghosts are states having negative norm. We have explained above that in order to construct the quantummechanical theory defined by a PT-symmetric Hamiltonian, we must construct the appropriate adjoint from the $C$ operator. Having constructed the $C P T$ adjoint, one may find that the so-called ghost state is actually not a ghost at all because when its norm is calculated using the appropriate definition of the adjoint, the norm turns out to be positive. This is what happens in the case of the Lee model.

The Lee model was proposed in 1954 as a quantum field theory in which mass, wavefunction, and charge reorganisation could be performed exactly and in closed form [47]. However, in 1955 Källén and Pauli showed that when the renormalised coupling constant is larger than a critical value, the Hamiltonian becomes non-Hermitian (in the Dirac sense) and a ghost state appears [44]. The importance of the work of Källén and Pauli was emphasised by Salam in his review of their paper [57] and the appearance of the ghost was assumed to be a fundamental defect of the Lee model. However, in 2005 it was shown that the non-Hermitian Lee-model Hamiltonian is PT symmetric and when the norms of the states of this model are determined using the $C$ operator, which can be calculated exactly and in closed form, the ghost state is seen to be an ordinary physical state having positive norm [9]. Thus, the following assertion by Barton [3] is not correct: "A non-Hermitian Hamiltonian is unacceptable partly because it may lead to complex energy eigenvalues, but chiefly because it implies a non-unitary S matrix, which fails to conserve probability and makes a hash of the physical interpretation."

Another example of a quantum model that was thought to have ghost states, but in fact does not, is the Pais-Uhlenbeck oscillator model [16, 17, 18]. This model has a fourth-order field equation, and for the past several decades it was thought (incorrectly) that all such higher-order field equations lead inevitably to ghosts. It is shown in Ref. [16] that when the Pais-Uhlenbeck model is quantised using the methods of PT quantum mechanics, it does not have any ghost states at all.

There are many potential applications for PT quantum mechanics in areas such as particle physics, cosmology, gravitation, quantum field theory, and solid-state physics. These applications are discussed in detail in the recent review article in Ref. [5]. Furthermore, there are now indications that theories described by $P T$-symmetric Hamiltonians can be observed in table-top experiments [48, 54, 55].

Having shown the validity and potential usefulness of PT quantum mechanics, one may 
ask why $P T$ quantum mechanics works. The reason is that $C P$ is a positive operator, and thus it can be written as the exponential of another operator $Q: C P=\mathrm{e}^{Q}$. The square root of $\mathrm{e}^{Q}$ can then be used to construct a new Hamiltonian $\tilde{H}$ via a similarity transformation on the $P T$-symmetric Hamiltonian $H: \tilde{H} \equiv \mathrm{e}^{-Q / 2} H \mathrm{e}^{Q / 2}$. The new Hamiltonian $\tilde{H}$ has the same energy eigenvalues as the original Hamiltonian $H$ because a similarity transformation is isospectral. Moreover, $\tilde{H}$ is Dirac Hermitian [51]; $P T$ quantum mechanics works because there is an isospectral equivalence between a non-Hermitian $P T$-symmetric Hamiltonian and a conventional Dirac Hermitian Hamiltonian.

There are a number of elementary examples of this equivalence, but a nontrivial illustration is provided by the Hamiltonian $H$ in (2) at $\epsilon=2$. This Hamiltonian is not Hermitian because boundary conditions that violate the $L^{2}$ norm must be imposed in Stokes wedges in the complex plane in order to obtain a real, positive, discrete spectrum. The exact equivalent Hermitian Hamiltonian is $\tilde{H}=p^{2}+4 x^{4}-2 \hbar x$, where $\hbar$ is Planck's constant [10, 23, 43]. The term proportional to $\hbar$ vanishes in the classical limit and is thus an example of a quantum anomaly.

Since $P T$ symmetry is equivalent by means of a similarity transformation to conventional Dirac Hermiticity, one may wonder whether PT quantum mechanics is actually fundamentally different from ordinary quantum mechanics. The answer is yes and, at least in principle, there is an experimentally observable difference between $P T$-symmetric and ordinary Dirac Hermitian Hamiltonians. The quantum brachistochrone provides a setting for examining this difference and provides a way to discriminate between the class of $P T$-symmetric Hamiltonians and the class of Dirac Hermitian Hamiltonians.

\section{COMPLEX CLASSICAL MOTION}

It is implicitly assumed in the derivation of the classical brachistochrone that the path of shortest time of descent is real. However, it is interesting that if one allows for the possibility of complex paths of motion, one can achieve an even shorter time of flight. In this section we consider a simple classical-mechanical system. Our purpose is to explain heuristically how extending a dynamical system into the complex domain can result in faster-than-real time evolution.

To demonstrate that a shorter time of flight can be achieved by means of complex paths, let us consider the classical harmonic oscillator, whose Hamiltonian is

$$
H=p^{2}+x^{2} .
$$

If a particle has energy $E=1$, then the classical turning points of the motion of the particle are located at $x= \pm 1$. The particle undergoes simple harmonic motion in which it oscillates sinusoidally between these two turning points. This periodic motion is indicated in Fig. 1 by a solid line connecting the turning points. However, in addition to this oscillatory motion on the real- $x$ axis, there are an infinite number of other trajectories that a particle of energy $E$ can have [7]. These classical trajectories, which are also shown in Fig. 1, are all ellipses whose foci are located at precisely the positions of the turning points. All of the classical orbits are periodic, and all orbits have the same period $T=2 \pi$. Thus, a classical particle travels faster along more distant ellipses.

Now suppose that a classical particle of energy $E=1$ travels along the real- $x$ axis from some point $x=-a$ to $x=a$, where $a>1$. If the potential $V(x)$ is everywhere zero along its 


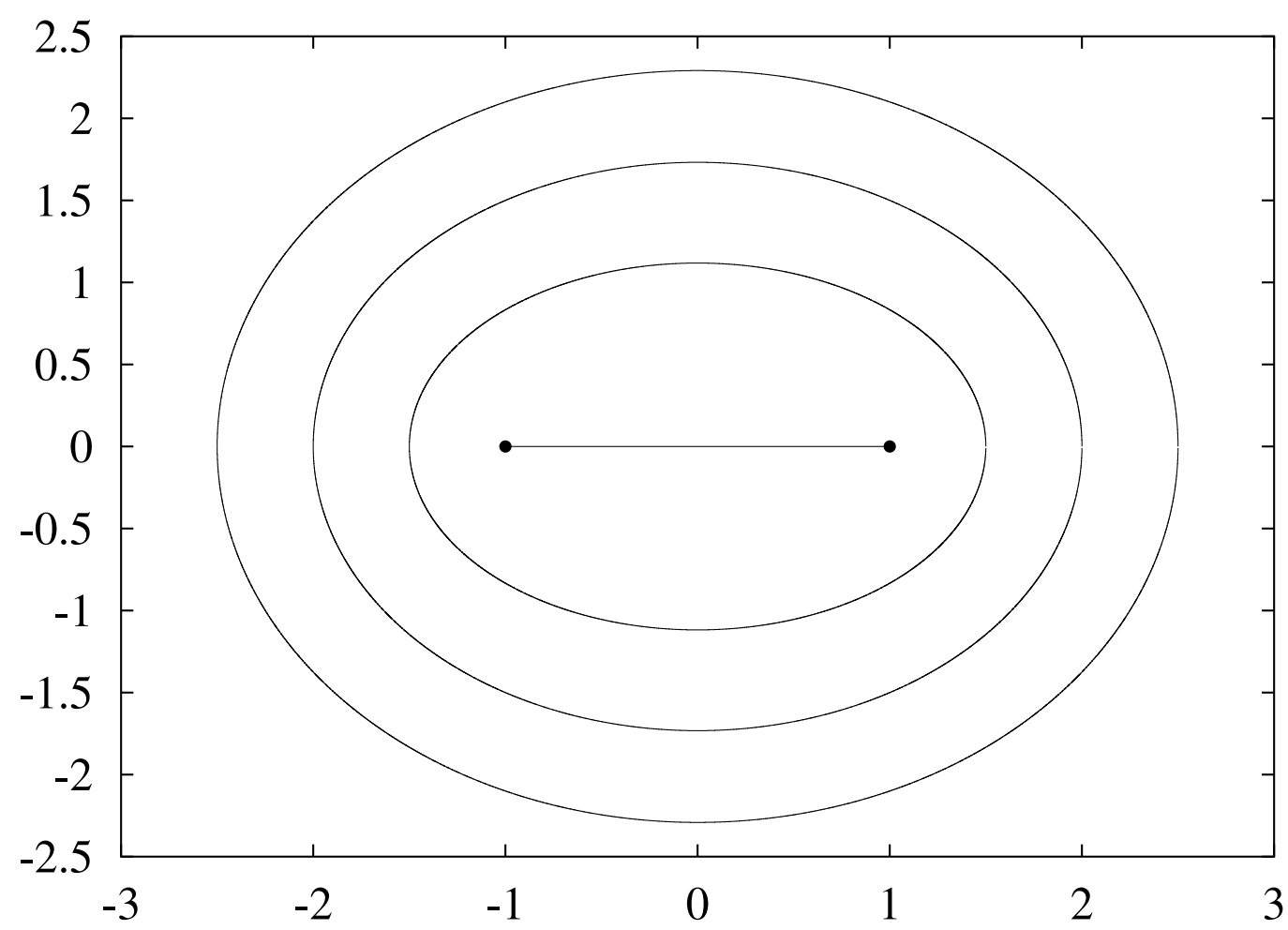

FIG. 1: Classical trajectories in the complex- $x$ plane for the harmonic oscillator whose Hamiltonian is $H=p^{2}+x^{2}$. These trajectories represent the possible paths of a particle whose energy is $E=1$. The trajectories are nested ellipses with foci located at the turning points at $x= \pm 1$. The real line segment (degenerate ellipse) connecting the turning points is the usual periodic classical solution to the harmonic oscillator. All closed paths have the same period $2 \pi$.

path, then it will travel at a constant velocity. However, suppose that the particle suddenly finds itself in the parabolic potential $V(x)=x^{2}$ when it reaches the turning point at $x=-1$ and that it suddenly escapes the influence of this potential at $x=1$. Then, the time of flight from $x=-a$ to $x=a$ will be changed because the particle does not travel at a constant velocity between the turning points. Next, let us imagine that the potential $V(x)=x^{2}$ is suddenly turned on before the particle reaches the turning point at $x=-1$. In this case, the particle will follow one of the elliptical paths in the complex plane around to the positive real axis. Just as the particle reaches the positive real axis the potential is turned off, so the particle proceeds onward along the real axis until it reaches $x=a$. This trip will take less time because the particle travels faster along the ellipse in the complex plane.

We have arrived at the surprising conclusion that if the classical particle enters the parabolic potential $V(x)=x^{2}$ immediately after it begins its voyage up the real axis, its time of flight will be exactly half a period, or $\pi$. Indeed, by travelling in the complex plane, a particle of energy $E=1$ can go from the point $x=-a$ to the point $x=a$ in time $\pi$, no matter how large $a$ is. Evidently, if a particle is allowed to follow complex classical trajectories, then it is possible to make a drastic reduction in its time of flight between two given real points. 


\section{HERMITIAN QUANTUM BRACHISTOCHRONE}

The quantum brachistochrone problem, as described briefly in Sec. I, is similar to the classical counterpart except that the optimisation takes place in a Hilbert space. Specifically, we are given a pair of quantum states, an initial state $\left|\psi_{I}\right\rangle$ and the final state $\left|\psi_{F}\right\rangle$, and we would like to find the one-parameter family of unitary operators $\left\{U_{t}\right\}$ that achieves the transformation $\left|\psi_{I}\right\rangle \rightarrow\left|\psi_{F}\right\rangle=U_{t}\left|\psi_{I}\right\rangle$ in the smallest possible time $t$. Since a oneparameter family of unitary operators can be formed in terms of a Hermitian operator $H$ as $U_{t}=\exp (-\mathrm{i} H t / \hbar)$, the problem is equivalent to finding the Hermitian operator $H$ that realises the transformation $\left|\psi_{I}\right\rangle \rightarrow\left|\psi_{F}\right\rangle$ in the shortest possible time.

The Hermitian operator $H$ can be thought of as representing the Hamiltonian, so the quantum brachistochrone problem is equivalent to finding the optimal Hamiltonian $H$ satisfying $\exp (-\mathrm{i} H t / \hbar)\left|\psi_{I}\right\rangle=\left|\psi_{F}\right\rangle$. However, it is intuitively clear that if we are allowed to have access to an unbounded energy resource, then the time required for the relevant transformation, irrespective of whether the Hamiltonian is optimal or not, can be made arbitrary small. Hence, for a quantum brachistochrone problem to possess a nontrivial solution, some form of constraint is needed. The simplest constraint is to assume that the energy is bounded so that the difference between the largest and the smallest energy eigenvalues has a fixed value $\omega$ :

$$
E_{\max }-E_{\min }=\omega
$$

A short calculation shows that if the Hamiltonian $H$ is bounded, then (i) the standard deviation of the energy is bounded according to

$$
\Delta H \leq \frac{1}{2}\left(E_{\max }-E_{\min }\right)
$$

and (ii) the state with maximum energy uncertainty is $\left(\left|E_{\max }\right\rangle+\left|E_{\min }\right\rangle\right) / \sqrt{2}$. It follows that the energy constraint (4) is equivalent to a constraint on energy uncertainty.

The brachistochrone problem of this type has been analysed recently and a solution was obtained by means of a variational method [26]. It has also been solved in terms of a more elementary approach making use of the geometry of quantum state space [20]. We shall follow closely the latter approach here.

Let us now state the simplest form of the quantum brachistochrone problem: We have a quantum system represented by an $N$-dimensional Hilbert space $\mathcal{H}$ and a prescribed pair of states $\left|\psi_{I}\right\rangle$ and $\left|\psi_{F}\right\rangle$ on $\mathcal{H}$. The problem is (a) to find the Hamiltonian $H$ satisfying the constraint (4) such that the unitary transformation $\exp (-\mathrm{i} H t / \hbar)\left|\psi_{I}\right\rangle=\left|\psi_{F}\right\rangle$ is achieved in shortest possible time and (b) to find the time required to realise such an operation.

A little geometric intuition allows us to find the solution to this problem with minimum effort. Recall that the time required for transporting a state along a path in $\mathcal{H}$ is given by the distance divided by the speed. Hence, all we have to do is first to identify the shortest path and measure its length and then to allow the state to evolve along the path with the greatest possible speed without violating the constraint (4).

In quantum mechanics the notion of distance is closely linked to the notion of transition probability [21, 42]. In particular, by looking at the transition probability between neighbouring states we can derive the expression for the metric on the space of quantum states. This allows us to measure distances between states. The idea can be sketched as follows: Consider a state $|\psi\rangle$ in $\mathcal{H}$ and a neighbouring state $|\psi\rangle+|\mathrm{d} \psi\rangle$. The transition probability 
between these states is

$$
\cos ^{2}\left(\frac{1}{2} \mathrm{~d} s\right)=\frac{(\langle\psi|+\langle\mathrm{d} \psi|) \mid \psi\rangle\langle\psi|(|\psi\rangle+|\mathrm{d} \psi\rangle)}{\langle\psi \mid \psi\rangle(\langle\psi|+\langle\mathrm{d} \psi|)(|\psi\rangle+|\mathrm{d} \psi\rangle)}
$$

where $\mathrm{d} s$ defines the line element on the space of pure states. By using

$$
\cos ^{2}\left(\frac{1}{2} \mathrm{~d} s\right) \approx 1-\frac{1}{4} \mathrm{~d} s^{2}
$$

expanding the right side of ([6), and retaining terms of quadratic order, we find that the line element is

$$
\mathrm{d} s^{2}=4 \frac{\langle\psi \mid \psi\rangle\langle\mathrm{d} \psi \mid \mathrm{d} \psi\rangle-\langle\psi \mid \mathrm{d} \psi\rangle\langle\mathrm{d} \psi \mid \psi\rangle}{\langle\psi \mid \psi\rangle^{2}} .
$$

This line element is known in geometry to arise from the Fubini-Study metric [46] and it can be used to measure the distance of the shortest path joining a pair of points on the space of pure quantum states.

If the Hilbert space is two dimensional, then a generic normalised state vector $|\psi\rangle$ can be expressed in the form

$$
|\psi\rangle=\left(\begin{array}{c}
\cos \frac{1}{2} \theta \\
\sin \frac{1}{2} \theta \mathrm{e}^{\mathrm{i} \phi}
\end{array}\right)
$$

A short calculation then shows that the Fubini-Study line element (8) reduces in this case to the expression

$$
\mathrm{d} s^{2}=\mathrm{d} \theta^{2}+\sin ^{2} \theta \mathrm{d} \phi^{2}
$$

which we recognise as the line element on the Bloch sphere $\mathcal{S}$. (The Bloch sphere is the state space of two-level systems.)

In the case of an $n$-dimensional Hilbert space $\mathcal{H}$, if we are given a pair of distinct states $\left|\psi_{I}\right\rangle$ and $\left|\psi_{F}\right\rangle$, then the linear span of these two states forms a two-dimensional subspace of $\mathcal{H}$. It should be intuitively clear that the shortest path joining $\left|\psi_{I}\right\rangle$ and $\left|\psi_{F}\right\rangle$ should lie on this two-dimensional subspace. Thus, irrespective of the dimensionality of $\mathcal{H}$ the solution to our quantum brachistochrone problem can be obtained by analysing the two-dimensional subspace spanned by $\left|\psi_{I}\right\rangle$ and $\left|\psi_{F}\right\rangle$. Even when we restrict our attention to this subspace, there still remain infinitely many unitary orbits that realise the transformation $\left|\psi_{I}\right\rangle \rightarrow\left|\psi_{F}\right\rangle=U_{t}\left|\psi_{I}\right\rangle$. However, since the two-dimensional state space is just the Bloch sphere $\mathcal{S}$ endowed with the spherical metric (10), we see that there is a unique great circle arc that joins $\left|\psi_{I}\right\rangle$ and $\left|\psi_{F}\right\rangle$ on $\mathcal{S}$. (This assumes, of course, that $\left|\psi_{I}\right\rangle$ and $\left|\psi_{F}\right\rangle$ are not antipodal points of the sphere. Otherwise, there are infinitely many such paths.) In this way we have identified the shortest path joining $\left|\psi_{I}\right\rangle$ and $\left|\psi_{F}\right\rangle$. The shortest distance $s_{\min }$ between these two points of $\mathcal{S}$ is thus given by

$$
s_{\min }=2 \arccos \left(\frac{\left|\left\langle\psi_{I} \mid \psi_{F}\right\rangle\right|}{\sqrt{\left\langle\psi_{I} \mid \psi_{I}\right\rangle\left\langle\psi_{F} \mid \psi_{F}\right\rangle}}\right) .
$$


This result can also be obtained by integrating the line element (10) along the great-circle $\operatorname{arc}$ on $\mathcal{S}$.

Having obtained the distance of the shortest path we proceed to find the maximum speed at which the state can evolve unitarily. For the evolution of the state we must consider the general Schrödinger equation, but we also need to express the equation in the correct form. This is the so-called modified Schrödinger equation

$$
\frac{\mathrm{d}\left|\psi_{t}\right\rangle}{\mathrm{d} t}=-\frac{\mathrm{i}}{\hbar} \tilde{H}\left|\psi_{t}\right\rangle .
$$

In this equation the mean-adjusted Hamiltonian $\tilde{H}$ is given by

$$
\tilde{H}=H-\langle H\rangle,
$$

where

$$
\langle H\rangle=\frac{\langle\psi|H| \psi\rangle}{\langle\psi \mid \psi\rangle} .
$$

Note that $\langle\tilde{H}\rangle=0$ and that according to (12) the tangent vector $\frac{\mathrm{d}}{\mathrm{d} t}\left|p s i_{t}\right\rangle$ is everywhere orthogonal to the direction of the state [45]. Since the energy expectation $\langle H\rangle$ depends on the state $|\psi\rangle$, the modified Schrödinger equation appears to be nonlinear. However, this is not the case. The point is that the expectation value of the Hamiltonian is a constant of the motion under the Schrödinger dynamics. Thus, given the initial state $\left|\psi_{I}\right\rangle$, we calculate $\langle H\rangle$ in this state and subtract this number from the Hamiltonian. Since the Hamiltonian in quantum mechanics is defined only up to an additive constant, this modification does not alter the physics in any way. It is worthwhile noting that the modified Schrödinger equation (12) is canonical and reduces to the standard eigenvalue problem when the state $\left|\psi_{t}\right\rangle$ is time independent without one having to evoke the correspondence principle [21].

If the initial state vector $\left|\psi_{I}\right\rangle$ is normalised, then the evolution (12) preserves the norm. It follows that $\langle\psi \mid \mathrm{d} \psi\rangle=0$. Since the speed $v$ of quantum evolution is given by $v=\mathrm{d} s / \mathrm{d} t$, we find from (81) and (12) that

$$
v^{2}=\frac{4}{\hbar^{2}}\left\langle\psi_{t}\left|(H-\langle H\rangle)^{2}\right| \psi_{t}\right\rangle=\frac{4}{\hbar^{2}}\left\langle\psi_{I}\left|(H-\langle H\rangle)^{2}\right| \psi_{I}\right\rangle .
$$

This shows that the speed of quantum evolution is given by the energy uncertainty. The expression (15) for the speed of quantum evolution is known as the Anandan-Aharonov relation [1]. Since we know from (5) that under the constraint (4) the energy uncertainty $\Delta H$ is bounded by $\frac{1}{2} \omega$, we find that the maximum speed of quantum evolution is given by

$$
v_{\max }=\frac{\omega}{\hbar} .
$$

By using the results in (11) and (16) we deduce that the minimum time required for realising the unitary transportation $\left|\psi_{I}\right\rangle \rightarrow\left|\psi_{F}\right\rangle=U_{t}\left|\psi_{I}\right\rangle$ is given by the ratio $s_{\min } / v_{\max }$. In particular, if $\left|\psi_{I}\right\rangle$ and $\left|\psi_{F}\right\rangle$ are orthogonal, then they correspond to antipodal points on the Bloch sphere $\mathcal{S}$, and we have $s_{\min }=\pi$. In this case, the minimum time required to orthogonalize the state (that is, for the state to evolve into a new state that is orthogonal to the original state) is known as the passage time $\tau_{\mathrm{P}}[19,58]$. The passage time is explicitly

$$
\tau_{\mathrm{P}}=\frac{\pi \hbar}{2 \Delta H}=\frac{\pi \hbar}{\omega} .
$$




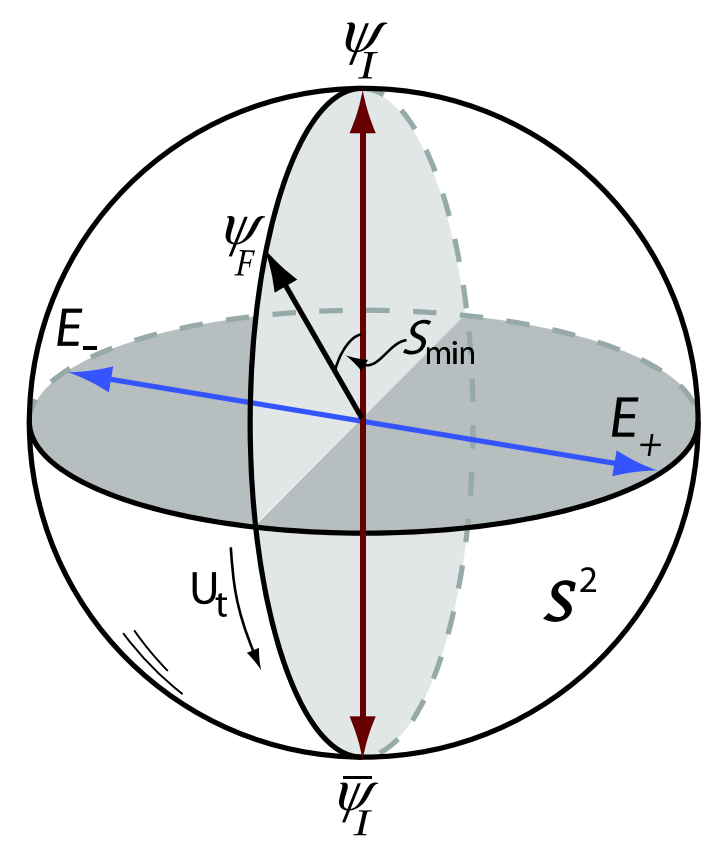

FIG. 2: Optimal Hamiltonian for quantum state transportation. The two-dimensional complex Hilbert space spanned by the initial state $\left|\psi_{I}\right\rangle$ and the final state $\left|\psi_{F}\right\rangle$ can be visualised in real terms as a Bloch sphere $\mathcal{S}$. The two states $\left|\psi_{I}\right\rangle$ and $\left|\psi_{F}\right\rangle$ can then be identified as a pair of points on $\mathcal{S}$. Assuming that these two points are not antipodal, there exists a unique great circle arc joining these two points, which determines the shortest path joining the two states. The optimal way of unitarily transporting $\left|\psi_{I}\right\rangle$ into $\left|\psi_{F}\right\rangle$ is therefore to rotate the sphere along the axis orthogonal to the great circle. The axis of rotation then specifies two quantum states: $\left|E_{-}\right\rangle$and $\left|E_{+}\right\rangle$. These states are the eigenstates of the optimal Hamiltonian $H$.

The passage time (17) provides the bound in Hermitian quantum mechanics for transporting a state into an orthogonal state, and is sometimes referred to as the Fleming bound [32].

The ratio $s_{\min } / v_{\max }$ gives the solution to part (b) of our quantum brachistochrone problem. To solve part (a), that is, to find the optimal Hamiltonian, we argue as follows: Since the problem is confined to a two-dimensional subspace of $\mathcal{H}$, the solution can be obtained by elementary trigonometry on the Bloch sphere $\mathcal{S}$. The key idea is to recall that the shortest path joining $\left|\psi_{I}\right\rangle$ and $\left|\psi_{F}\right\rangle$ is a great circle arc on $\mathcal{S}$. Without loss of generality, we can assume that $\left|\psi_{I}\right\rangle$ and $\left|\psi_{F}\right\rangle$ lie on the equator of $\mathcal{S}$ with respect to a suitable choice of axis. Then, the unitary motion along the shortest path can be generated by the rotation of $\mathcal{S}$ along this axis. Since the eigenstates of the Hamiltonian $H$ that generates such a rotation correspond to the antipodal points along this axis, the pair of states $\left|\psi_{I}\right\rangle$ and $\left|\psi_{F}\right\rangle$ can both be expressed as equal superpositions of the eigenstates of $H$ with the relative phase shifted by $s_{\text {min }}$. Writing $\left|E_{+}\right\rangle$and $\left|E_{-}\right\rangle$for the normalised eigenstates of $H$ and using $\alpha=s_{\min } / 2$, we can express the initial and the final state in the form

$$
\frac{1}{\sqrt{ } 2}\left(\left|E_{-}\right\rangle+\mathrm{e}^{-\mathrm{i} \alpha}\left|E_{+}\right\rangle\right)=\left|\psi_{I}\right\rangle \quad \text { and } \quad \frac{1}{\sqrt{ } 2}\left(\left|E_{-}\right\rangle+\mathrm{e}^{\mathrm{i} \alpha}\left|E_{+}\right\rangle\right)=\left|\psi_{F}\right\rangle .
$$

Solving these equations for $\left|E_{+}\right\rangle$and $\left|E_{-}\right\rangle$, we obtain

$$
\left|E_{-}\right\rangle=\frac{\mathrm{i}}{\sqrt{ } 2 \sin \alpha}\left(\mathrm{e}^{-\mathrm{i} \alpha}\left|\psi_{F}\right\rangle-\mathrm{e}^{\mathrm{i} \alpha}\left|\psi_{I}\right\rangle\right)
$$


and

$$
\left|E_{+}\right\rangle=-\frac{\mathrm{i}}{\sqrt{ } 2 \sin \alpha}\left(\left|\psi_{F}\right\rangle-\left|\psi_{I}\right\rangle\right) .
$$

These are the eigenstates of the optimal Hamiltonian $H$ that generates the unitary motion $\left|\psi_{I}\right\rangle \rightarrow\left|\psi_{F}\right\rangle=U_{t}\left|\psi_{I}\right\rangle$ along the shortest path. The eigenvalues of the optimal $H$ can be arbitrary as long as the condition (4) is satisfied. Without loss of generality, we may assume $H$ be trace free, and we obtain the solution to part (a) of the quantum brachistochrone problem:

$$
H=\frac{1}{2} \omega\left|E_{+}\right\rangle\left\langle E_{+}\left|-\frac{1}{2} \omega\right| E_{-}\right\rangle\left\langle E_{-}\right| .
$$

This is the 'minimal' solution to the problem in the sense that $H$ acts only on the twodimensional subspace of $\mathcal{H}$ while leaving the rest of $\mathcal{H}$ unperturbed.

As a special example, consider the problem of a spin-flip, that is, turning a spin-up state into a spin-down state unitarily. In this case the initial and the final states can be written as

$$
\left|\psi_{I}\right\rangle=\left(\begin{array}{l}
1 \\
0
\end{array}\right) \text { and }\left|\psi_{F}\right\rangle=\left(\begin{array}{l}
0 \\
1
\end{array}\right)
$$

in the spin- $z$ basis. Substituting these into (19) and (20), we find that the eigenstates of the optimal Hamiltonian are

$$
\left|E_{-}\right\rangle=\frac{1}{\sqrt{ } 2}\left(\begin{array}{l}
1 \\
1
\end{array}\right) \quad \text { and } \quad\left|E_{+}\right\rangle=\frac{\mathrm{i}}{\sqrt{ } 2}\left(\begin{array}{c}
1 \\
-1
\end{array}\right)
$$

because in this case we have $\alpha=\pi / 2$. Substituting this result into (21) yields

$$
H=\frac{1}{2}\left(\begin{array}{cc}
0 & -\omega \\
-\omega & 0
\end{array}\right)
$$

for the optimal Hamiltonian. By using the relation

$$
\mathrm{e}^{\mathrm{i} \phi \sigma \cdot \mathbf{n}}=\cos \phi \mathbb{1}+\mathrm{i} \sin \phi \sigma \cdot \mathbf{n},
$$

where $\mathbf{n}$ is a unit vector and

$$
\sigma_{1}=\left(\begin{array}{cc}
0 & 1 \\
1 & 0
\end{array}\right), \quad \sigma_{2}=\left(\begin{array}{cc}
0 & -i \\
i & 0
\end{array}\right), \quad \sigma_{3}=\left(\begin{array}{cc}
1 & 0 \\
0 & -1
\end{array}\right)
$$

are Pauli matrices, we obtain the expression for the optimal unitary operator:

$$
U_{t}=\left(\begin{array}{rr}
\cos \left(\frac{\omega t}{2 \hbar}\right) & -\mathrm{i} \sin \left(\frac{\omega t}{2 \hbar}\right) \\
-\mathrm{i} \sin \left(\frac{\omega t}{2 \hbar}\right) & \cos \left(\frac{\omega t}{2 \hbar}\right)
\end{array}\right) .
$$

It follows at once that the optimal unitary orbit $\left|\psi_{t}\right\rangle=U_{t}\left|\psi_{I}\right\rangle$ is given by

$$
\left|\psi_{t}\right\rangle=\left(\begin{array}{c}
\cos \left(\frac{\omega t}{2 \hbar}\right) \\
-\mathrm{i} \sin \left(\frac{\omega t}{2 \hbar}\right)
\end{array}\right)
$$


We find that the first time at which $\left|\psi_{t}\right\rangle$ reaches $\left|\psi_{F}\right\rangle$ is given by the condition $\omega t / 2 \hbar=\pi / 2$, that is, when $t=\tau_{\mathrm{P}}$, where $\tau_{\mathrm{P}}$ is the passage time given in (17).

We have seen how the simplest form of a quantum brachistochrone problem can be solved in Hermitian quantum mechanics by considering a two-dimensional Hilbert subspace combined with elementary geometric constructions on it. In a more general situation the unitary motion may be constrained further so that the optimal Hamiltonian (21) may not be implementable. For example, the constraint may enforce the path of the unitary evolution to lie in a three- rather than in a two-dimensional subspace. To determine what happens let us work out the passage time for this example.

Since in this case the minimal solution $H$ to the brachistochrone problem is a threedimensional matrix, we can express the initial state $\left|\psi_{I}\right\rangle$ in terms of the three eigenstates of $H$ according to

$$
\left|\psi_{I}\right\rangle=\cos \alpha\left|E_{i}\right\rangle+\sin \alpha \cos \beta \mathrm{e}^{\mathrm{i} \phi}\left|E_{j}\right\rangle+\sin \alpha \sin \beta \mathrm{e}^{\mathrm{i} \varphi}\left|E_{k}\right\rangle
$$

where $\alpha, \beta$ are angular coordinates, $\phi, \varphi$ are phase variables, and we assume that $E_{i}<$ $E_{j}<E_{k}$. If a unitary operator $U_{T}$ transforms this state into an orthogonal state, then the condition

$$
\cos ^{2} \alpha+\sin ^{2} \alpha \cos ^{2} \beta \mathrm{e}^{-\mathrm{i} \omega_{j i} T / \hbar}+\sin ^{2} \alpha \sin ^{2} \beta \mathrm{e}^{-\mathrm{i} \omega_{k i} T / \hbar}=0
$$

must be satisfied, where $\omega_{j i}=E_{j}-E_{i}$ and $\omega_{k i}=E_{k}-E_{i}$. To render the analysis more tractable, we simplify this constraint by assuming that $\alpha=\beta=\pi / 4$. Then (30) implies that a necessary condition for the state $\left|\psi_{I}\right\rangle$ to evolve into an orthogonal state is given by the relation

$$
\frac{\omega_{k i}}{\omega_{j i}}=\frac{2 m-1}{2 n-1}
$$

where $m, n$ are natural numbers such that $m \neq n$.

Thus, the solution to the brachistochrone problem must be such that the eigenvalues of $H$ satisfy condition (31) as well as the constraint $E_{\max }-E_{\min } \leq \omega$. Assuming that these constraints are indeed satisfied, the initial state evolves into an orthogonal state $\left|\psi_{F}\right\rangle$. The first time that $\left|\psi_{I}\right\rangle$ evolves into a state orthogonal to $\left|\psi_{I}\right\rangle$, in particular, is given by

$$
T=\frac{\pi \hbar}{\omega_{j i}}=\frac{3 \pi \hbar}{\omega_{k i}}
$$

However, since in this case $U_{t}\left|\psi_{I}\right\rangle$ does not describe a geodesic path, $T$ will be larger than Fleming's passage time $\tau_{\mathrm{P}}$ given in (17). Indeed, without loss of generality we may set $E_{i}=0$. Then, it is straightforward to verify that $T=\sqrt{6} \tau_{\mathrm{P}}$. This follows from the fact that under the constraint $\omega_{k i}=3 \omega_{j i}$ that comes from (32), the squared energy dispersion in the state (29) with $\alpha=\beta=\pi / 4$ is given by $\Delta H^{2}=\frac{3}{2} \omega_{j i}^{2}$.

\section{NON-HERMITIAN QUANTUM BRACHISTOCHRONE}

We have seen how the solution to the simple brachistochrone problem can be obtained in the Hermitian quantum theory. What happens if we extend the quantum theory into the complex domain by looking at a PT-symmetric theory? We saw earlier that in classical 
mechanics if we were to allow for a complex path interpolating a pair of real points of the coordinate space, then it is possible (at least mathematically) to transport a particle across a large distance in virtually no time. It turns out that an analogous situation emerges in the $P T$-symmetric theory. Here we present a simple algebraic calculation of the optimal evolution time from an initial state to a final state by using a simple $2 \times 2$ Hamiltonian. As we have remarked above, the $2 \times 2$ model suffices to cover general cases because in the case of our simple brachistochrone problem the solution is found on the two-dimensional subspace of the Hilbert space spanned by the initial state $\left|\psi_{I}\right\rangle$ and the final state $\left|\psi_{F}\right\rangle$. In the case of a $P T$-symmetric Hamiltonian the variational approach gives a more direct way to handle the brachistochrone problem. Thus, we shall first briefly revisit the Hermitian case but expressed in the variational formalism and then we will compare the result to its $P T$-symmetric counterpart.

\section{A. Hermitian $2 \times 2$ matrices}

We choose a basis so that the initial and final state vectors take the form

$$
\left|\psi_{I}\right\rangle=\left(\begin{array}{l}
1 \\
0
\end{array}\right) \quad \text { and }\left|\psi_{F}\right\rangle=\left(\begin{array}{c}
a \\
b
\end{array}\right),
$$

where the condition that $\left|\psi_{F}\right\rangle$ be normalised is $|a|^{2}+|b|^{2}=1$. The most general $2 \times 2$ Hermitian Hamiltonian is

$$
H=\left(\begin{array}{cc}
s & r \mathrm{e}^{-\mathrm{i} \theta} \\
r \mathrm{e}^{\mathrm{i} \theta} & u
\end{array}\right) \quad(r, s, u, \theta \text { real }) .
$$

For this Hamiltonian the eigenvalue constraint (4) takes the form

$$
\omega^{2}=(s-u)^{2}+4 r^{2} .
$$

To find the optimal Hamiltonian satisfying this constraint, we rewrite $H$ as a linear combination of Pauli matrices:

$$
H=\frac{1}{2}(s+u) \mathbb{1}+\frac{1}{2} \omega \sigma \cdot \mathbf{n},
$$

where

$$
\mathbf{n}=\frac{1}{\omega}(2 r \cos \theta, 2 r \sin \theta, s-u)
$$

is a unit vector. Then by use of the identity (25) the evolution equation $\left|\psi_{F}\right\rangle=\mathrm{e}^{-\mathrm{i} H \tau / \hbar}\left|\psi_{I}\right\rangle$ can be expressed in the form

$$
\left(\begin{array}{l}
a \\
b
\end{array}\right)=\mathrm{e}^{-\frac{1}{2} \mathrm{i}(s+u) t / \hbar}\left(\begin{array}{c}
\cos \frac{\omega t}{2 \hbar}-\mathrm{i} \frac{s-u}{\omega} \sin \frac{\omega t}{2 \hbar} \\
-\mathrm{i} \frac{2 r}{\omega} \mathrm{e}^{\mathrm{i} \theta} \sin \frac{\omega t}{2 \hbar}
\end{array}\right) .
$$

The second component of this equation gives $|b|=\frac{2 r}{\omega} \sin \frac{\omega t}{2 \hbar}$, which allows us to find the required time of evolution:

$$
t=\frac{2 \hbar}{\omega} \arcsin \frac{\omega|b|}{2 r} .
$$


We must now minimise the time $t$ over all $r>0$ while maintaining the energy constraint in (35). This constraint tells us that the maximum value of $r$ is $\frac{1}{2} \omega$. At this value we have $s=u$. Because $H$ can be made trace free, we can set $s=u=0$. The variable $\theta$ in (36) does not affect the eigenvalues, so we may set $\theta=\pi$. Then we recover the optimal Hamiltonian obtained in (24). As regards the minimum evolution time $\tau$ we have

$$
\tau=\frac{2 \hbar \arcsin |b|}{\omega}
$$

In the special case for which $a=0$ and $b=1$ so that $\left|\psi_{I}\right\rangle$ and $\left|\psi_{F}\right\rangle$ are orthogonal, we recover the passage time $\tau=\tau_{\mathrm{P}}=\pi \hbar / \omega$, the smallest time required for a spin flip.

Although the form of the result in (40) resembles the statement of the uncertainty principle, it is merely the statement indicated above that rate $\times$ time $=$ distance; the maximum speed of evolution is given by $\Delta H$, and the distance between $\left|\psi_{I}\right\rangle$ and $\left|\psi_{F}\right\rangle$, assuming they are normalised, is given by $2 \arccos \left(\left|\left\langle\psi_{F} \mid \psi_{I}\right\rangle\right|\right)$. Since $\left|\left\langle\psi_{F} \mid \psi_{I}\right\rangle\right|=|a|$ and $|a|=\sqrt{1-|b|^{2}}$, we obtain (40) from the relation

$$
\arccos \sqrt{1-|b|^{2}}=\arcsin |b|
$$

\section{B. Non-Hermitian $2 \times 2$ matrices}

We now show by direct calculation that for a $P T$-symmetric Hamiltonian, $\tau$ can be arbitrarily small. This is because a $P T$-symmetric Hamiltonian whose eigenvalues are all real is equivalent to a Hermitian Hamiltonian via $\tilde{H}=\mathrm{e}^{-Q / 2} H \mathrm{e}^{Q / 2}$, where $Q$ is Dirac Hermitian. The states in a $P T$-symmetric theory are mapped by $\mathrm{e}^{-Q / 2}$ to the corresponding states in the Dirac Hermitian theory. But, the overlap distance between two states does not remain constant under a similarity transformation. We can exploit this property of the similarity transformation to overcome the Hermitian lower limit on the time $\tau$. (The detailed calculation is explained in Ref. [14].)

We consider the general class of $P T$-symmetric $2 \times 2$ Hamiltonians having the form

$$
H=\left(\begin{array}{cc}
r \mathrm{e}^{\mathrm{i} \theta} & s \\
s & r \mathrm{e}^{-\mathrm{i} \theta}
\end{array}\right) \quad(r, s, \theta \text { real }) .
$$

The time reversal operator $T$ performs complex conjugation and the parity operator in this case is given by

$$
P=\left(\begin{array}{ll}
0 & 1 \\
1 & 0
\end{array}\right)
$$

The two eigenvalues

$$
E_{ \pm}=r \cos \theta \pm \sqrt{s^{2}-r^{2} \sin ^{2} \theta}
$$

are real if $s^{2}>r^{2} \sin ^{2} \theta$. This inequality defines the region of unbroken $P T$ symmetry. The unnormalised eigenstates of $H$ are

$$
\left|E_{+}\right\rangle=\left(\begin{array}{c}
\mathrm{e}^{\mathrm{i} \alpha / 2} \\
\mathrm{e}^{-\mathrm{i} \alpha / 2}
\end{array}\right) \text { and }\left|E_{-}\right\rangle=\left(\begin{array}{c}
\mathrm{ie} \\
-\mathrm{i} \mathrm{e}^{\mathrm{i} \alpha / 2} / 2
\end{array}\right)
$$


where $\alpha$ is given by $\sin \alpha=(r / s) \sin \theta$. Note that the condition of unbroken $P T$ symmetry of $H$ in (42) implies that $\alpha$ is real. The $C$ operator required for defining the Hilbert space inner product is

$$
C=\frac{1}{\cos \alpha}\left(\begin{array}{cc}
\mathrm{i} \sin \alpha & 1 \\
1 & -\mathrm{i} \sin \alpha
\end{array}\right)
$$

It is easy to verify that the CPT norms of both eigenstates have the value $\sqrt{2 \cos \alpha}$.

To calculate $\tau$ we express the Hamiltonian $H$ in (42) as

$$
H=(r \cos \theta) \mathbb{1}+\frac{1}{2} \omega \sigma \cdot \mathbf{n},
$$

where

$$
\mathbf{n}=\frac{2}{\omega}(s, 0, i r \sin \theta)
$$

is a unit vector. The energy constraint requires that the squared difference between energy eigenvalues is

$$
\omega^{2}=4 s^{2}-4 r^{2} \sin ^{2} \theta
$$

The positivity of $\omega^{2}$ is ensured by the condition of unbroken PT symmetry. Notice that (49) differs from (35) by a sign. We can think of (49) as being hyperbolic in character, while (35) is elliptic in character. The technical advantage of the constraint in (49) is that because of the minus sign both terms on the right side can become large without violating the condition that $\omega$ be fixed. We will see that it is this fact that allows the non-Hermitian Hamiltonian $H$ in (42) to achieve faster-than-Hermitian time evolution.

To determine $\tau$ we write down the $P T$-symmetric time-evolution equation in vector form:

$$
\mathrm{e}^{-\mathrm{i} H t / \hbar}\left(\begin{array}{l}
1 \\
0
\end{array}\right)=\frac{\mathrm{e}^{-\mathrm{i} t r \cos \theta / \hbar}}{\cos \alpha}\left(\begin{array}{c}
\cos \left(\frac{\omega t}{2 \hbar}-\alpha\right) \\
-\mathrm{i} \sin \left(\frac{\omega t}{2 \hbar}\right)
\end{array}\right)
$$

In particular, consider the pair of vectors used in the Hermitian spin-flip case as in (22). Observe that the evolution time $t$ needed to reach $\left|\psi_{F}\right\rangle=\left(\begin{array}{l}0 \\ 1\end{array}\right)$ from $\left|\psi_{I}\right\rangle=\left(\begin{array}{l}1 \\ 0\end{array}\right)$ is given by

$$
t=\frac{(2 \alpha-\pi) \hbar}{\omega}
$$

Optimising this result over allowable values for $\alpha$ as $\alpha$ approaches $\frac{1}{2} \pi$, the optimal time $\tau$ tends to zero, a dramatic change from the Hermitian result in (17)! Note, however, that the two vectors in (22) are not orthogonal with respect to the CPT inner product. This is the reason that the Fleming bound in (17) is not violated.

\section{EXTENSION OF NON-HERMITIAN HAMILTONIANS TO HIGHER-DIMENSIONAL HERMITIAN HAMILTONIANS}

We have seen how a quantum state can be transported unitarily into another state in arbitrary short time by using a bounded Hamiltonian if we allow for a complex path interpolating them in the space of unitary motions. Can such an operation be implemented 
in practice? If the answer is affirmative, then the implication is immense in quantum information, computation, cryptography, and other related fields. For example, if a quantum computer were to exist, then solutions to difficult optimisation problems can in principle be found in arbitrary short time, and this in turn would have important implications in society as a whole.

A gedanken experiment was proposed in Ref. [14] to realise this effect in a laboratory. The setup is as follows: we use a Stern-Gerlach filter to create a beam of spin-up electrons. The beam then passes through a black box containing a device governed by a PT-symmetric Hamiltonian that flips the spins unitarily in a very short time. The outgoing beam then enters a second Stern-Gerlach device that verifies that the electrons are now in spin-down states. In effect, the black box device is applying a complex magnetic field $\vec{B}$ :

$$
\vec{B}=(s, 0, \mathrm{i} r \sin \theta) \text {. }
$$

If the field strength has sufficiently large amplitude, then spins can be flipped in virtually no time because the complex path joining these two states is arbitrarily short without violating the energy constraint in (49). We emphasise that the field strength can be made large without violating the energy constraint (44) is a consequence of the hyperbolic representation in (49).

The arbitrarily short alternative complex pathway from an up state to a down state, as illustrated by this thought experiment, is reminiscent of the short alternative distance between two widely separated space-time points as measured through a wormhole in general relativity. This comparison is of course controversial, and it has subsequently motivated much research and it has generated a lively debate in the literature [2, 15, 33, 35, 36, 49, 52, 53, 56]. We emphasise that the entire package of flipping the spin is not realised by a unitary operation. This follows from the fact that $P T$-symmetric quantum theory is unitary, and as such it respects the Fleming bound (17) applicable to all unitary theories [14]. The point is that the 'black box' scheme described above actually consists of three regimes: (i) the preparation of a spin-up state in the Hermitian setup, (ii) the fast unitary motion to flip the spin using a PT-symmetric Hamiltonian, and (iii) the recovery of a spindown state in the Hermitian setup. Thus, the operation is locally unitary, but the switching between Hermitian and PT-symmetric description is not unitary. This three-step process of switching Hamiltonians is analogous to the classical procedure described in Sec. III for obtaining faster-than-real time evolution. Recall that in the classical case we were able to transport a finite-energy particle across a large distance in a short time by switching the potential through which it was travelling. Note that in the classical case there is no question of violating unitarity because the particle does not get lost.

The question of unitarity and faster-than-Hermitian time evolution has been reexamined in more detail in Ref. [35, 53] by means of a geometric approach and also in Ref. [2], where a more general class of non-Hermitian Hamiltonians that are not necessarily $P T$ symmetric are considered. In particular, Mostafazadeh has emphasised the role of quantum observables in such an experiment; the spin operator in Hermitian quantum mechanics cannot be interpreted as a spin operator in the $P T$-symmetric counterpart, thus leading to ambiguities regarding the physical interpretation of the gedanken experiment described above.

An intriguing alternative proposal for an implementation of the fast spin flip has been made more recently by Günther and Samsonov [36]. The idea is to embed the problem into a Hermitian setup represented by a higher-dimensional Hilbert space. Specifically, take our 
$P T$-symmetric Hamiltonian $H$ in (42). The eigenstates of $H$ are not orthogonal with respect to the Hermitian inner product. Since $H$ is not Hermitian, its Hermitian conjugate defines a new matrix $H^{\dagger}$. The eigenstates of $H^{\dagger}$ thus also define a pair of nonorthogonal states in the Hermitian theory. When these four states are suitably normalised, they can be used to form an over-complete basis set in the Hermitian two-dimensional Hilbert space. Such an over-complete set of basis is also known as a positive operator-valued measure (POVM), commonly used in the analysis of quantum information theory. A key idea is that such a basis can be embedded in a higher-dimensional Hilbert space to form an orthogonal basis by using the Naimark dilation [40]. A Hermitian Hamiltonian can then be constructed - in this case a $4 \times 4$ matrix - such that its eigenstates are precisely the four states thus obtained. Using this Hamiltonian it is possible to construct a standard unitary motion in such a way that the induced motion obtained by the projection onto the two-dimensional subspace is characterised by the $P T$-symmetric motion (50).

In this way, Günther and Samsonov were able to show that the fast spin flip can in principle be realised in the standard Hermitian quantum mechanics by a combination of a unitary motion and a projection in a larger-dimensional Hilbert space. In practical terms this means that one should couple the spin to an auxiliary particle (this can be done either by a projection or by a unitary operation), apply a unitary evolution in the larger Hilbert space of the combined system, and finally project out the auxiliary particle to recover the spin in the transported state. The net effect of such an operation can then be characterised by (50). The Fleming bound is apparently violated due to the general fact that when a unitary motion is projected to a subspace of a Hilbert space, the resulting dynamics need not respect laws of unitarity. It would be of considerable interest to find out whether the Günther and Samsonov scheme can actually be implemented in a laboratory, and if not, what might be the difficulty preventing the violation of the Fleming bound.

\section{Acknowledgments}

We have benefited greatly from many discussions with Drs. U. Günther and B. Samsonov. We thank Dr. D. W. Hook for his assistance in preparing the figures used in this chapter. CMB is supported by a grant from the U.S. Department of Energy.

[1] Anandan, J. and Aharonov, Y.: Geometry of quantum Eeolution. Phys. Rev. Lett. 65, 1697$1700(1990)$

[2] Assis, P. and Fring, A.: The quantum brachistochrone problem for non-Hermitian Hamiltonians. J. Phys. A: Math. Theor. 41, 244002 (12 pages) (2008)

[3] Barton, G.: Introduction to Advanced Field Theory (John Wiley \& Sons, New York, 1963), chap. 12

[4] Bender, C. M.: Introduction to PT-symmetric quantum theory. Contemp. Phys. 46, 277-292 (2005)

[5] Bender, C. M.: Making sense of non-Hermitian Hamiltonians. Rep. Prog. Phys. 70, 947-1018 (2007)

[6] Bender, C. M. and Boettcher, S.: Real spectra in Nnn-Hermitian Hamiltonians having PT symmetry. Phys. Rev. Lett. 80, 5243-5246 (1998) 
[7] Bender, C. M., Boettcher, S., and Meisinger, P. N.: PT-symmetric quantum mechanics. J. Math. Phys. 40, 2201-2209 (1999)

[8] Bender, C. M., Brandt, S. F., Chen, J.-H., and Wang, Q.: The $C$ operator in PT-symmetric quantum field theory transforms as a Lorentz scalar. Phys. Rev. D 71, 065010 (7 pages) (2005)

[9] Bender, C. M., Brandt, S. F., Chen, J.-H., and Wang, Q.: Ghost busting: PT-Symmetric Interpretation of the Lee Model. Phys. Rev. D 71, 025014 (11 pages) (2005)

[10] Bender, C. M., Brody, D. C., Chen, J.-H., Jones, H. F., Milton, K. A., and Ogilvie, M. C.: Equivalence of a complex PT-symmetric quartic Hamiltonian and a Hermitian quartic Hamiltonian with an anomaly. Phys. Rev. D 74, 025016 (10 pages) (2006)

[11] Bender, C. M., Brody, D. C., and Jones, H. F.: Complex extension of quantum mechanics. Phys. Rev. Lett. 89, 270401 (4 pages) (2002)

[12] Bender, C. M., Brody, D. C., and Jones, H. F.: Must a Hamiltonian be Hermitian? Am. J. Phys. 71, 1095-1102 (2003)

[13] Bender, C. M., Brody, D. C., and Jones, H. F.: Scalar quantum field theory with complex cubic interaction. Phys. Rev. Lett. 93, 251601 (4 pages) (2004)

[14] Bender, C. M., Brody, D. C., Jones, H. F., and Meister, B. K.: Faster than Hermitian quantum mechanics. Phys. Rev. Lett. 98, 040403 (4 pages) (2007)

[15] Bender, C. M., Brody, D. C., Jones, H. F., and Meister, B. K.: Comment on the quantum brachistochrone problem. arXiv:0804.3487 [quant-ph]

[16] Bender, C. M. and Mannheim, P. D.: No-ghost theorem for the fourth-order derivative PaisUhlenbeck oscillator model. Phys. Rev. Lett. 100, 110402 (4 pages) (2008)

[17] Bender, C. M. and Mannheim, P. D.: Exactly solvable PT-symmetric Hamiltonian having no Hermitian counterpart. Phys. Rev. D 78, 025022 (17 pages) (2008)

[18] Bender, C. M. and Mannheim, P. D.: Giving up the Ghost. J. Phys. A: Math. Theor. 41, 304018 (7 pages) (2008)

[19] Brody, D. C.: Elementary derivation of passage times. J. Phys. A: Math. Gen. 36, 5587-5593 (2003)

[20] Brody, D. C. and Hook, D. W.: On optimum Hamiltonians for state transformations. J. Phys. A: Math. Gen. 39, L167-L170 (2006). [Corrigendum. Ibid. 40, 10949 (2007)]

[21] Brody, D. C. and Hughston, L. P.: Geometric quantum mechanics. J. Geom. Phys. 38, 19-53 (2001)

[22] Brower, R., Furman, M., and Moshe, M.: Critical exponents for the Reggeon quantum spin model. Phys. Lett. B 76, 213-219 (1978)

[23] Buslaev, V. and Grecchi, V.: Equivalence of unstable anharmonic oscillators and double wells. J. Phys. A: Math. Gen. 26, 5541-5549 (1993)

[24] Cardy, J. L.: Conformal invariance and the Yang-Lee edge singularity in two dimensions. Phys. Rev. Lett. 54, 1354-1356 (1985)

[25] Cardy, J. L. and Mussardo, G.: S-matrix of the Yang-Lee edge singularity in two dimensions. Phys. Lett. B 225, 275-278 (1989)

[26] Carlini, A., Hosoya, A., Koike, T., and Okudaira, Y.: Time-optimal quantum evolution. Phys. Rev. Lett. 96, 060503 (4 pages) (2006)

[27] Dorey, P., Dunning, C., and Tateo, R.: Supersymmetry and the spontaneous breakdown of PT symmetry. J. Phys. A: Math. Gen. 34, L391-L400 (2001) 
[28] Dorey, P., Dunning, C., and Tateo, R.: Spectral equivalences, Bethe ansatz equations, and reality properties in PT-symmetric quantum mechanics. J. Phys. A: Math. Gen. 34, 5679-5704 (2001)

[29] Dorey, P., Dunning, C., and Tateo, R.: The ODE/IM correspondence. J. Phys. A: Math. Theor. 40, R205-R283 (2007)

[30] Faria, C. F. de M. and Fring, A.: Non-Hermitian Hamiltonians with real eigenvalues coupled to electric fields: from the time-independent to the time dependent quantum mechanical formulation. Laser Phys. 17, 424-437 (2007)

[31] Fisher, M. E.: Yang-Lee edge singularity and $\phi^{3}$ field theory. Phys. Rev. Lett. 40, 1610-1613 (1978)

[32] Fleming, G. N.: A unitary bound on the evolution of nonstationary states. Nuov. Cim. A 16, $232(1973)$

[33] Günther, U., Rotter, I., and Samsonov, B. F.: Projective Hilbert space structures at exceptional points. J. Phys. A: Math. Theor. 40, 8815-8833 (2007)

[34] Günther, U., Samsonov, B. F., and Stefani, F.: A globally diagonalizable $\alpha^{2}$-dynamo operator, SUSY QM and the Dirac equation. J. Phys. A: Math. Theor. 40, F169-F176 (2007)

[35] Günther, U. and Samsonov, B. F.: The PT-symmetric brachistochrone problem, Lorentz boosts and non-unitary operator equivalence classes. arXiv: 0709.0483 [quant-ph]

[36] Günther, U. and Samsonov, B. F.: The Naimark dilated PT-symmetric brachistochrone. arXiv:0807.3643

[37] Guenther, U., Stefani, F., and Znojil, M.: MHD $\alpha^{2}$-dynamo, Squire equation and PTsymmetric interpolation between square well and harmonic oscillator. J. Math. Phys. 46, 063504 (22 pages) (2005)

[38] Harms, B., Jones, S., and Tan, C.-I: New structure in the energy spectrum of Reggeon quantum mechanics with quartic couplings. Phys. Lett. B 91, 291-295 (1980)

[39] Harms, B., Jones, S., and Tan, C.-I: Complex energy spectra in Reggeon quantum mechanics with quartic interactions. Nucl. Phys. B 171, 392-412 (1980)

[40] Holevo, A. S.: Probabilistic and Statistical Aspects of Quantum Theory (North-Holland, Amsterdam, 1982)

[41] Hollowood, T.: Solitons in affine Toda field theories. Nucl. Phys. B 384, 523-540 (1992)

[42] Hughston, L. P.: Geometric aspects of quantum mechanics. In Twistor Theory, ed. by S. Huggett (Marcel Dekker, New York, 1995)

[43] Jones, H. F. and Mateo, J.: Equivalent Hermitian Hamiltonian for the non-Hermitian $-x^{4}$ potential. Phys. Rev. D 73, 085002 (4 pages) (2006)

[44] Källén, G. and Pauli, W.: On the mathematical structure of T. D. Lee's model of a renormalizable field theory. Dansk Vid. Selsk. Mat.-Fys. Medd. 30, No. 7, 23 pages (1955)

[45] Kibble, T. W. B.: Geometrization of quantum mechanics. Commun. Math. Phys. 65, 189-201 (1979)

[46] Kobayashi, S. and Nomizu, K.: Foundations of Differential Geometry, Vol. 2 (Wiley, New York, 1969)

[47] Lee, T. D.: Some special examples in renormalizable field theory. Phys. Rev. 95, 1329-1334 (1954) 
[48] Makris, K. G., El-Ganainy, R., Christodoulides, D. N., and Musslimani, Z. H.: Beam dynamics in PT symmetric optical lattices. Phys. Rev. Lett. 100, 103904 (4 pages) (2008)

[49] Martin, D.: Is PT-symmetric quantum mechanics just quantum mechanics in a non-orthogonal basis? arXiv: quant-ph/0701223v2

[50] Mostafazadeh, A.: Pseudo-Hermiticity versus PT-symmetry III. J. Math. Phys. 43, 3944-3951 (2002)

[51] Mostafazadeh, A.: Exact PT-symmetry is equivalent to Hermiticity. J. Phys. A: Math. Gen. 36 (2003), 7081-7091.

[52] Mostafazadeh, A.: Quantum brachistochrone problem and the geometry of the state space in pseudo-Hermitian quantum mechanics. Phys. Rev. Lett. 99, 130502 (4 pages) (2007)

[53] Mostafazadeh, A.: Physical meaning of Hermiticity and shortcomings of the composite (Hermitian + non-Hermitian) quantum theory of G" unther and Samsonov. arXiv:0709.1756 [quantph]

[54] Musslimani, Z. H., Makris, K. G., El-Ganainy, R., and Christodoulides, D. N.: Analytical solutions to a class of nonlinear Schrödinger equations with PT-like potentials. J. Phys. A: Math. Theor. 41, 244019 (12 pages) (2008)

[55] Musslimani, Z. H., Makris, K. G., El-Ganainy, R., and Christodoulides, D. N.: Optical solitons in PT periodic potentials. Phys. Rev. Lett. 100, 030402 (4 pages) (2008)

[56] Rotter, I.: The brachistochrone problem in open quantum systems. J. Phys. A: Math. Theor. 40 14515-14526 (2007)

[57] Salam, A.: Review of On the mathematical structure of T. D. Lee's model of a renormalizable field theory by G. Källén and W. Pauli, MathSciNet Mathematical Reviews on the Web MR0076639 (17,927d) (1956)

[58] Schulman, L. S.: Jump Time and Passage Time. In Time in Quantum Mechanics, ed. by J. G. Muga, R. Sala Mayato, and I. L. Egusquiza (Springer-Verlag, Berlin, 2002)

[59] Wigner, E. P.: Phenomenological distinction between unitarity and antiunitarity symmetry operators. J. Math. Phys. 1, 414-416 (1960)

[60] Wu, T. T.: Ground state of a Bose system of hard spheres. Phys. Rev. 115, 1390-1404 (1959)

[61] Zamolodchikov, A. B.: Two-point correlation function in scaling Lee-Yang model. Nucl. Phys. B 348, 619-641 (1991)

[62] Another way to see that $T$ is associated with complex conjugation is to require that the time-dependent Schrödinger equation be invariant under time reversal. This implies that time reflection $t \rightarrow-t$ must be accompanied by complex conjugation $\mathrm{i} \rightarrow-\mathrm{i}$. See Ref. [59] for a discussion of the properties of antilinear operators. 